\title{
Perinatal and neonatal predictors of developmental coordination disorder in very low birthweight children
}

\author{
Jill Glennis Zwicker, ${ }^{1,2}$ Shin Won Yoon, ${ }^{3}$ Margot MacKay, ${ }^{4}$ Julie Petrie-Thomas, ${ }^{4}$ \\ Marilyn Rogers, ${ }^{4}$ Anne R Synnes ${ }^{1,2,4}$
}

\begin{abstract}
${ }^{1}$ Department of Pediatrics, University of British Columbia, Vancouver, Canada

${ }^{2}$ Developmental Neuroscience and Child Health, Child \&

Family Research Institute, Vancouver, Canada

${ }^{3}$ Department of Pediatrics, National Health Insurance Corporation, Ilsan Hospital, Koyang, Korea

${ }^{4}$ Neonatal Follow-Up Program, British Columbia's Women's Hospital, Vancouver, Canada
\end{abstract}

\section{Correspondence to}

Dr Anne R Synnes, Neonatal Follow-Up Program, British Columbia's Women's Hospital, Room 1R13, 4480 Oak St., Vancouver, BC, Canada V6H 3V4; asynnes@cw.bc.ca

Received 29 April 2012 Revised 17 September 2012 Accepted 28 November 2012

\section{ABSTRACT \\ Objective To identify perinatal and neonatal risk factors associated with developmental coordination disorder $(D C D)$ in very low birthweight children \\ (VLBW: <1250 g). \\ Design Retrospective design with prospectively collected cohort. \\ Setting Neonatal Follow-Up Program, Vancouver, Canada. \\ Patients 157 VLBW children assessed at 4-5 years who were free of cerebral palsy or major neurological impairment and had full-scale IQ $>70$.}

Main outcome measure Movement Assessment Battery for Children (MABC).

Results Using $\leq 15$ th percentile on the MABC as the cut-off, $42 \%$ of our cohort developed DCD. Perinatal variables significantly associated with $D C D$ were male sex, lower gestational age and lower birth weight, but only male sex and low birth weight independently predicted $D C D$, accounting for $20 \%$ of the variance in $M A B C$ scores. Compared with children without motor impairment, children with DCD had greater postnatal steroid exposure, longer duration of ventilation, more days on oxygen and significant retinopathy of prematurity, but only postnatal steroid exposure was significant, accounting for an additional $3 \%$ of the variance in $\mathrm{MABC}$ scores. Boys performed more poorly than girls on all subtests of the MABC.

Conclusions Male sex and low birth weight were significant predictors of $D C D$, suggesting that these infants should be followed for detection of this common, but under-recognised disorder. Future research aimed at identifying neural underpinnings of DCD and possible antecedents to the disorder is warranted.

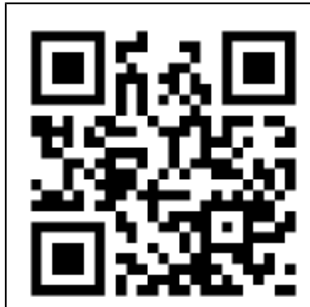

Scan to access more free content

\footnotetext{
To cite: Zwicker JG, Yoon SW, MacKay M, et al. Arch Dis Child

2013;98:118-122.
}

\section{INTRODUCTION}

Developmental coordination disorder (DCD) is a motor disorder of unknown aetiology that significantly interferes with a child's ability to perform motor daily tasks (eg, tying shoes, riding a bicycle, printing). According to the Diagnostic and Statistical Manual, 4 th edition, ${ }^{1}$ there are four diagnostic criteria for DCD: (A) motor coordination substantially below expected for a person's chronological age and measured intelligence; (B) the motor disturbance significantly interferes with academic achievement or activities of daily living; (C) the disturbance is not due to a general medical condition (eg, cerebral palsy); and (D) the motor difficulties are in excess of those usually associated with the person's intelligence. DCD can have a considerable impact on the quality of life of children, including limited participation in physical

\section{What is already known on this topic}

- Children born preterm are at high risk to develop developmental coordination disorder.

- Current research has reported variable findings in terms of perinatal and neonatal risk factors for the disorder in the preterm population.

\section{What this study adds}

- We confirm that male sex, very low birth weight and postnatal steroids are significant risk factors for developmental coordination disorder (DCD).

- Using the European Academy of Childhood Disability guidelines for $D C D$, over $40 \%$ of very premature infants may meet diagnostic criteria for the disorder.

- We demonstrate that $D C D$ can be identified at or before school entry, showing the potential for early identification and intervention.

and social activities, problems with peers, and emotional health concerns. ${ }^{2}$ While DCD affects approximately $5 \%$ of school-age children, ${ }^{1}$ children born very premature ( $\leq 32$ weeks gestational age) are 6-8 times more likely to develop the disorder. ${ }^{3}$

Several studies have explored possible perinatal and neonatal factors associated with DCD in the premature population with inconsistent results. Holsti $e t ~ a l^{4}$ found no difference in several neonatal variables, including chronic lung disease, patent ductus arteriosus (PDA), necrotising enterocolitis, duration of ventilation or days on oxygen between children of extremely low birth weight $(\leq 800 \mathrm{~g})$ who did and did not develop DCD. Goyen and $\mathrm{Lui}^{5}$ found that prolonged rupture of membranes (PROM) and retinopathy of prematurity (ROP) were significantly associated with DCD in extremely premature $(\leq 29$ weeks) or extremely low birthweight children $(\leq 1000 \mathrm{~g})$ at age 8 years. In a similar cohort of extremely low birth weight infants at school-age, Davis et $a l^{6}$ found male sex and postnatal steroid exposure to be associated with DCD, whereas Cooke $^{7}$ found that only low gestational age was associated with poor motor outcomes consistent with DCD in a cohort of very premature infants. Despite the high prevalence of DCD in premature infants, ${ }^{3}$ no consistent pattern 
of perinatal and neonatal factors have emerged to explain the relationship of DCD in this vulnerable population. Identifying perinatal risk factors of DCD may help generate hypotheses regarding aetiology and help identify those children at the highest risk.

The purpose of our study was to evaluate the association of perinatal and neonatal risk factors with DCD in a cohort of very low birthweight $(\leq 1250 \mathrm{~g})$ children followed prospectively in our Neonatal Follow-Up Program (NFUP). Given that previous studies have identified DCD at 7-9 years of age, ${ }^{4-7}$ our secondary aim was to identify and describe the presentation of DCD in premature infants at an earlier age, before or soon after school entry. Identifying children at risk of developing motor difficulties at an earlier age may help to initiate intervention and minimise secondary complications.

\section{METHODS}

\section{Study population}

Children (ages 4-5 years) seen at the NFUP at the British Columbia's Women's Hospital between May 2005 and October 2009 were eligible to participate in the study $(n=221)$. NFUP recruitment criteria include birth weight $\leq 800 \mathrm{~g}$, gestational age $\leq 25$ completed weeks, grade 4 intraventricular haemorrhage as per Papile et al, ${ }^{8}$ cystic periventricular leukomalacia, severe ROP ( $\geq$ stage 3 or requiring laser treatment), home oxygen therapy or participants in funded research studies. Most NFUP participants are cared for in the adjacent Neonatal Intensive Care Unit, which has approximately 600 admissions annually, 16\%-19\% of them infants weighing less than $1250 \mathrm{~g}$ at birth.

Inclusion criteria for this study were children seen in the NFUP with birth weights $<1250 \mathrm{~g}$ who completed the Movement Assessment Battery for Children (MABC) ${ }^{9}$ at age 4 years 6 months to 5 years 11 months. Children were excluded if they were diagnosed with cerebral palsy as defined by Bax, ${ }^{10}$ had significant cognitive delay (defined as a full-scale IQ (FSIQ) score $<70$ ) or were blind (defined as acuity in the better eye with refractive correction worse than 20/200). This study was approved by the University of British Columbia and British Columbia's Women's Hospital Research Ethics Board. Parents consented to the NFUP data being used for research purposes.

\section{Clinical data}

We retrospectively obtained perinatal and neonatal clinical data from review of discharge summaries from the Neonatal Intensive Care Unit. Nine perinatal variables were extracted: any exposure to antenatal steroids, PROM greater than $18 \mathrm{~h}$, mode of delivery (vaginal vs Caesarean), singleton or multiple birth, sex, 5 min Apgar score, gestational age, birth weight, and birth weight for gestational age expressed as an SD score using local growth curves (unpublished data, Whitfield, 1993). Neonatal information included: days of supplemental oxygen, days of ventilation, exposure to postnatal steroids, presence or absence of clinically significant PDA, medical or surgical treatment for PDA, history of necrotizing enterocolitis ROP (defined as grade 3 or 4 ROP or requiring laser treatment), history of sepsis (positive blood culture), hyponatraemia (sodium $<130$ milliequivalents per litre) and cranial ultrasound abnormalities.

\section{Outcomes}

Children were evaluated at 4-5 years of age by a multidisciplinary team, including occupational therapists, physicians and psychologists. The MABC, ${ }^{9}$ a common assessment used to identify children with DCD ${ }^{11}$ was administered by an occupational therapist using a standard protocol. The MABC has eight tasks, grouped in three sections: manual dexterity (three tasks), ball skills (two tasks) and balance skills (three tasks). Each individual task is scored between 0 and 5, for a total impairment score ranging from 0 to 40 . Higher scores indicate poorer performance. The total impairment score was transformed to a percentile rank using the MABC manual. Consistent with the recently published European Academy of Childhood Disability guidelines, ${ }^{12}$ we used a score of $\leq 15$ th percentile on the MABC as our definition of DCD. Because previous guidelines recommended a score of $\leq 5$ th percentile to classify children with DCD ${ }^{13}$ we divided our sample into children with scores $\leq 5$ th percentile (severe DCD) and scores between the 6th and 15 th percentile (mild-moderate DCD). Scores $>15$ th percentile were classified as non-DCD.

\section{Data analysis}

Statistical analyses were performed using IBM SPSS Statistics, V.19 (IBM, New York, USA). Differences between perinatal and neonatal risk factors by DCD group were calculated using Pearson $\chi^{2}$ test for categorical variables and one-way analyses of variance (ANOVA) for continuous data. As the variances of MABC scores were not homogeneous between the groups (Levene statistic, $\mathrm{p}<0.001$ ), we conducted Welch and BrownForsythe tests to confirm that we could use ANOVA results (both $\mathrm{p}<0.001$ ). Statistical significance was set at $\mathrm{p}<0.05$. Statistically significant variables were entered into a two-step logistic regression model (first incorporating perinatal and then neonatal variables) to examine the association of these factors with MABC scores. When two variables were highly correlated (variance inflation factor $(\mathrm{VIF})>10$ ), the variable of greater statistical significance in the univariable analyses was entered into the model. We also examined sex differences at 4-5 years using Fisher's exact test for categorical variables and one-way ANOVA for continuous data.

\section{RESULTS}

Of the 221 children with birth weights $<1250 \mathrm{~g}$ who were assessed at age 4-5 years in the NFUP between May 2005 and October 2009, the MABC was completed on 179 (81\%). After excluding children with cerebral palsy $(n=11)$ and those with a FSIQ $<70 \quad(n=11)$, our final sample size was 157 children (79 boys; 78 girls). As shown in table 1, 45 children (29\%) scored $\leq 5$ th percentile on the MABC and were classified as severe DCD. With an additional 20 children (13\%) scoring in the mild-moderate DCD range, $42 \%$ of the cohort had DCD using the definition of MABC $\leq 15$ percentile.

Perinatal and neonatal characteristics for the three DCD groups are shown in table 1 . Perinatal variables significantly associated with DCD were male sex, lower gestational age and lower birth weight. Antenatal steroid exposure, PROM, type of delivery and Apgar scores were not significantly associated with DCD in our cohort. Neonatal factors significantly associated with DCD were postnatal steroid exposure, longer duration of ventilation, more days on oxygen, ROP and hyponatraemia. We found no significant relationship of treated PDA, sepsis, necrotizing enterocolitis and abnormal cranial ultrasound with DCD outcome.

Significant perinatal factors from the corrected univariable analyses were entered into the first step of the regression analysis. Given the high collinearity between gestational age and birth weight (VIF $>10$ ), birth weight (the more significant variable in univariable analyses) was entered into the model. Male sex and low birth weight were independently and significantly related to MABC scores, accounting for $20 \%$ of the variance 
Table 1 Perinatal and neonatal variables according to DCD group

\begin{tabular}{|c|c|c|c|c|}
\hline & Severe DCD & Mild-Moderate DCD & Non-DCD & p Value \\
\hline$n=157$ & 45 & 20 & 92 & \\
\hline MABC (percentile) & $2.3(1.7)$ & $9.4(2.6)$ & $43.7(22.2)$ & $<0.001$ \\
\hline Median (range) & $1(1-5)$ & $9(6-15)$ & $38(16-93)$ & \\
\hline Full-scale IQ & $89.0(11.7)$ & $96.9(14.3)$ & $101.0(13.2)$ & $<0.001$ \\
\hline Median (range) & $91(70-118)$ & $96.5(71-124)$ & $99(71-132)$ & \\
\hline \multicolumn{5}{|l|}{ Perinatal variables } \\
\hline Gestational age (weeks) & $25.5(1.8)$ & $26.8(2.0)$ & $26.5(1.7)$ & 0.004 \\
\hline Birth weight (grams) & 722 (169) & 814 (169) & 882 (195) & $<0.001$ \\
\hline Weight SD score & $-0.5(1.4)$ & $-0.8(0.9)$ & $-0.2(1.2)$ & 0.07 \\
\hline Male & $32(71)$ & $10(50)$ & $37(40)$ & 0.003 \\
\hline Antenatal steroid exposure & $31(69)$ & $17(85)$ & $77(86)^{*}$ & 0.06 \\
\hline $\mathrm{PROM}>18 \mathrm{~h}$ & $10(22)$ & $9(45)$ & $26(28)$ & 0.17 \\
\hline Caesarean delivery & $30(67)$ & $14(70)$ & $56(61)^{*}$ & 0.76 \\
\hline Singleton birth & $33(73)$ & $16(80)$ & $62(68) \dagger$ & 0.53 \\
\hline 5 min Apgar $<7$ & $11(25) \dagger$ & $5(25)$ & $16(18) \ddagger$ & 0.57 \\
\hline \multicolumn{5}{|l|}{ Neonatal variables } \\
\hline Postnatal steroid exposure & $20(44)$ & $7(35)$ & $15(16) \dagger$ & 0.002 \\
\hline Ventilation (days) & $50(25)$ & $32(28)$ & $31(21)$ & $<0.001$ \\
\hline Oxygen (days) & $100(57)$ & $74(83)$ & $60(55)$ & 0.002 \\
\hline ROP therapy & $19(42)$ & $3(15)$ & $14(15)$ & 0.001 \\
\hline Hyponatraemia & $26(58)$ & $6(30)$ & $30(33)$ & 0.01 \\
\hline PDA treated & $28(62)$ & $9(45)$ & $48(52)$ & 0.37 \\
\hline Sepsis & $25(56)$ & $10(50)$ & $52(57) \dagger$ & 0.84 \\
\hline Necrotising enterocolitis & $3(7)$ & $0(0)$ & $7(8) \dagger$ & 0.44 \\
\hline Abnormal cranial ultrasound & $22(49)$ & $6(30)$ & 34 (37) & 0.26 \\
\hline PVL or IPH & $4(9)$ & $2(10)$ & $8(9)$ & 0.98 \\
\hline Grade I-II IVH & $15(33)$ & $4(20)$ & $22(24)$ & 0.40 \\
\hline Grade III-IV IVH & $3(7)$ & $0(0)$ & $3(3)$ & 0.39 \\
\hline
\end{tabular}

Data are presented as number (\%) for categorical variables and mean (SD) for continuous variables, unless otherwise stated.

${ }^{*}$ Missing two values.

tMissing one value.

¥Missing three values.

DCD, developmental coordination disorder; IPH, intraparenchymal haemorrhage; IVH, intraventricular haemorrhage; MABC, Movement Assessment Battery for Children; PDA, patent ductus arteriosus; PROM, prolonged rupture of membranes; PVL, periventricular leukomalacia; ROP, retinopathy of prematurity.

(table 2). Significant neonatal variables were then added to the model: postnatal steroid exposure, ventilation days, days on oxygen, ROP and hyponatraemia. Male sex and low birth weight remained significant with the addition of neonatal factors, but postnatal steroid exposure was the only significant neonatal variable, accounting for an additional $3 \%$ of the variance in MABC scores.

Significantly more boys $(42 / 79 ; 53 \%)$ than girls $(24 / 78 ; 31 \%)$ scored in the DCD range of $\leq 15$ th percentile on the MABC $(p=0.006)$. Boys weighed significantly more for gestational age and had significantly lower Apgar scores than girls $(\mathrm{p}=0.03$ and 0.05 , respectively). Boys and girls did not differ across other perinatal or any neonatal variables (table 3 ). Boys performed worse than girls on all subtests of the MABC, but both genders performed in a similar pattern, with the poorest performance on balance subtests, followed by manual dexterity and then ball skills (data not shown).

\section{DISCUSSION}

\section{DCD is common in children born preterm}

Our study, performed in an era of surfactant and antenatal steroid use, confirms that DCD remains a common condition among children born very preterm. Our cohort cannot be generalised to all premature infants with birth weights $<1250 \mathrm{~g}$ because of the selective nature of our recruitment criteria (selection bias). However, the $42 \%$ incidence of DCD, using a more liberal definition of $\leq 15$ th percentile on the MABC, does reflect a regional population and is comparable with the incidence of

Table 2 Perinatal and neonatal predictors of developmental coordination disorder

\begin{tabular}{lcccc}
\hline Perinatal model & Coefficient & SE & $\mathbf{p ~ V a l u e ~}$ & Adjusted $\mathbf{R}^{2}$ \\
\hline Birth weight & 0.002 & 0.00 & $<0.001$ & 0.20 \\
Male sex & -0.51 & 0.13 & $<0.001$ & \\
$\begin{array}{l}\text { First perinatal and neonatal model } \\
\quad 0.001\end{array}$ & 0.00 & 0.007 & 0.23 \\
$\quad$ Birth weight & -0.47 & 0.13 & $<0.001$ & \\
Male sex & -0.30 & 0.16 & 0.06 & \\
Postnatal steroids & -0.001 & 0.004 & 0.88 & \\
Ventilation days & -0.001 & 0.001 & 0.60 & \\
Oxygen days & -0.190 & 0.17 & 0.26 & \\
Retinopathy of prematurity & & & & \\
Final perinatal and neonatal model & 0.001 & 0.00 & $<0.001$ & 0.23 \\
Birth weight & -0.50 & 0.13 & $<0.001$ & \\
Male sex & -0.38 & 0.15 & 0.01 & \\
Postnatal steroids & & & & \\
\hline
\end{tabular}


Table 3 Perinatal and neonatal variables by sex

\begin{tabular}{|c|c|c|c|}
\hline & Boys $(n=79)$ & Girls $(n=78)$ & $p$ Value \\
\hline \multicolumn{4}{|l|}{ Perinatal variables } \\
\hline Gestational age (weeks) & $26.0(1.6)$ & $26.4(2.0)$ & 0.15 \\
\hline Birth weight (grams) & $837(194.1)$ & $817(199.8)$ & 0.53 \\
\hline Weight SD score & $-0.16(1.3)$ & $-0.58(1.1)$ & 0.03 \\
\hline Antenatal steroid exposure & $67(86)^{*}$ & $58(75)^{*}$ & 0.11 \\
\hline $\mathrm{PROM}>18 \mathrm{~h}$ & $27(34)$ & $18(23)$ & 0.16 \\
\hline Caesarean delivery & $52(67)^{*}$ & $48(62)^{*}$ & 0.62 \\
\hline Singleton birth & $57(73)^{*}$ & $54(69)$ & 0.72 \\
\hline 5 min Apgar $<7$ & $21(28) \dagger$ & $11(14)^{*}$ & 0.05 \\
\hline \multicolumn{4}{|l|}{ Neonatal variables } \\
\hline Postnatal steroid exposure & $21(27)$ & $21(27)^{*}$ & 0.99 \\
\hline Ventilation (days) & $37(23.7)$ & $36(25.8)$ & 0.77 \\
\hline Oxygen (days) & $78(59.4)$ & $69(63.9)$ & 0.37 \\
\hline Retinopathy of prematurity & $19(24)$ & $17(22)$ & 0.85 \\
\hline Hyponatraemia & $35(44)$ & $27(35)$ & 0.25 \\
\hline PDA treated & $45(57)$ & $40(51)$ & 0.52 \\
\hline Sepsis & $43(54)$ & $44(57)^{*}$ & 0.75 \\
\hline Necrotising enterocolitis & $6(8)$ & $4(5)^{*}$ & 0.75 \\
\hline Abnormal cranial ultrasound & $35(44)$ & $27(35)$ & 0.25 \\
\hline PVL or IPH & $9(11)$ & $5(6)$ & 0.40 \\
\hline Grade I-II IVH & $24(30)$ & $17(22)$ & 0.28 \\
\hline Grade III-IV IVH & $2(3)$ & $4(5)$ & 0.44 \\
\hline
\end{tabular}

$40.5 \%$ reported in a recent meta-analysis of several studies reporting motor outcomes of preterm children. ${ }^{14}$ DCD is, therefore, a common condition in very low birthweight children which will be missed if developmental screening is not continued beyond 3 years. Given the multiple quality of life domains that may be affected by DCD in childhood and beyond, ${ }^{2}$ early identification and intervention are the key to improving the developmental outcomes in this vulnerable population.

\section{Perinatal variables of male sex and very low birth weight are significantly associated with DCD}

Perinatal variables of male sex, low gestational age and low birth weight differed significantly between children with and without DCD. As previously noted, male $\operatorname{sex}^{6}$ and lower birth weight $^{3}{ }^{14}$ significantly predicted DCD in our preterm cohort. Given the high collinearity between gestational age and birth weight, it is not surprising that others have found an association between low gestational age and DCD in the preterm population. ${ }^{3}$ In contrast to Goyen and Lui, ${ }^{5}$ we did not find that PROM was significantly associated with DCD.

\section{Neonatal variables are less associated with DCD}

DCD groups differed in several neonatal variables, including postnatal steroid exposure, duration of ventilation, days on oxygen and significant ROP (grade 3 or 4 or laser treated). Consistent with Davis et $a l,{ }^{6}$ we found that children with DCD had significantly more exposure to postnatal steroids than children who did not develop the disorder. Postnatal steroid exposure was the only significant neonatal predictor of DCD, which is consistent with other research showing postnatal steroids, such as dexamethasone, being associated with poor motor outcome. ${ }^{15}$ Because we collected postnatal steroid exposure as a binary variable, the potential relationship of amount of exposure (dose and duration) of postnatal steroids to DCD could not be explored.

Consistent with Goyen and Lui, ${ }^{5}$ ROP was significantly more prevalent in children with DCD in our cohort; however, treated ROP did not significantly predict DCD outcome. Although hyponatraemia was not a significant predictor of DCD, low sodium was significantly more apparent in children with severe DCD; this finding is consistent with other research showing poorer MABC scores in formerly premature school-age children with neonatal hyponatraemia. ${ }^{16}$

\section{Gender differences between preterm children with and with DCD}

DCD was significantly more prevalent in boys compared with girls, a finding consistent with clinical samples of children with DCD. ${ }^{17}$ Boys performed more poorly than girls on all subtests of the MABC, but both genders produced a similar pattern of scores. Balance appeared to be most severely affected, followed by dexterity. Thus, poor balance and fine-motor skills may be 'early' markers for DCD.

\section{Cranial ultrasound abnormalities did not predict DCD}

In preterm newborns, cranial ultrasound abnormalities indicative of white matter damage are strong predictors of cerebral palsy and developmental delay. ${ }^{18}$ Our study excluded children with severe neurodevelopmental disability (cerebral palsy, FSIQ < 70) and therefore reduced the numbers of subjects with periventricular leukomalacia or grade $4 \mathrm{IVH}$. This may explain why our study did not show any correlations between DCD and abnormal cranial ultrasound findings. However, DCD may be related to a milder spectrum of injury that may not be seen on cranial ultrasound or even on conventional MRI. ${ }^{19}$ The few neuroimaging studies that have been conducted in children with DCD show no 'overt' signs of injury, but rather altered patterns of brain activation ${ }^{20-23}$ and altered microstructural integrity of motor and sensory pathways. ${ }^{24}$ Linking advanced neuroimaging techniques, such as diffusion tensor imaging, of the premature brain and motor outcomes may shed some light on the possible mechanisms of DCD in the preterm population. Given the hypothesised role of the cerebellum in $\mathrm{DCD}^{25}$ and the altered cerebellar development in the preterm brain, ${ }^{26-28}$ further exploration of the relationship of cerebellar development and motor outcomes in the preterm population is warranted.

\section{Limitations}

This study is limited by its retrospective design, in that we could only gather data that was systematically documented in the medical chart; thus, other possible perinatal and neonatal variables that may predict DCD could not be explored. While our sample may have been heterogeneous in terms of the reasons for follow-up, we obtained a similar incidence of DCD in comparison with that reported in a meta-analysis of several studies of premature newborns. ${ }^{14}$ In addition, we were able to identify preterm children with DCD at an earlier age than other published studies. Due to the lack of a standardised method to identify the impact of motor problems on activities of daily living in the preschool-age population, we were unable to formally assess criterion $B$ of the DCD diagnostic criteria. ${ }^{1}$ However, we stringently ruled out other medical diagnoses (eg, CP), obtained a measure of IQ, and used a common, standardised measure of motor impairment to fulfil 
other diagnostic criteria for DCD. In the mild-moderate DCD group, three participants had IQs between -1 and -2 SDs and criterion D could not be definitively determined. We retained these children in the sample because of the recognition that 'a specific IQ level does not seem to be helpful to distinguish between children with DCD and children with coordination problems due to mental retardation, ${ }^{12}$ (p. 63).

\section{CONCLUSIONS}

In a cohort of 157 children born with birth weights $<1250 \mathrm{~g}$, we identified $42 \%$ as having DCD at age $4-5$ years. Male sex, low birth weight and postnatal steroid exposure were significant predictors of DCD, suggesting that these infants should be followed for detection of this common, but under-recognised disorder. Future research aimed at identifying neural underpinnings of DCD and possible antecedents to the disorder is warranted.

Acknowledgements We extend our thanks to the children and families who participated and the members of the Neonatal Follow-Up Program who organised the appointments and performed the clinical assessments of the participants reported in this study.

Contributors All authors have made substantive intellectual contributions to the study and have met the following criteria for authorship: ARS and SWY designed the study; SWY, MM, JPT and MR contributed to data collection; JGZ, ARS and JPT analysed and interpreted the data. JGZ drafted the article and all other authors revised it critically for intellectual content. All authors have approved the final version of the manuscript.

Funding Dr Zwicker is funded by the Canadian Child Health Clinician Scientist Program, Child and Family Research Institute, Michael Smith Foundation for Health Research, and NeuroDevNet.

Competing interests None.

Provenance and peer review Not commissioned; externally peer reviewed.

\section{REFERENCES}

1 American Psychiatric Association. Diagnostic and statistical manual of mental disorders. 4th edn. text revision. Washington, DC: Author, 2000

2 Zwicker JG, Harris SR, Klassen AF. Quality of life domains affected in children with developmental coordination disorder: a systematic review. Child Care Health Dev 2012 Apr 20. doi: 10.1111/j.1365-2214.2012.01379.x. [Epub ahead of print].

3 Edwards J, Berube M, Erlandson K, et al. Developmental coordination disorder in school-aged children born very preterm and/or at very low birth weight: a systematic review. J Dev Behav Pediatr 2011;32:678-87.

4 Holsti L, Grunau RV, Whitfield MF. Developmental coordination disorder in extremely low birth weight children at nine years. J Dev Behav Pediatr 2002;23:9-15.

5 Goyen TA, Lui K. Developmental coordination disorder in 'apparently normal' schoolchildren born extremely preterm. Arch Dis Child 2009:94:298-302.

6 Davis NM, Ford GW, Anderson PJ, et al. Developmental coordination disorder at 8 years of age in a regional cohort of extremely-low-birthweight or very preterm infants. Dev Med Child Neurol 2007;49:325-30.
7 Cooke RW. Perinatal and postnatal factors in very preterm infants and subsequent cognitive and motor abilities. Arch Dis Child Fetal Neonatal Ed 2005:90:F60-3.

8 Papile LA, Burstein J, Burstein R, et al. Incidence and evolution of subependymal and intraventricular hemorrhage: a study of infants with birth weights less than 1500 gm. J Pediatr 1978;92:529-34.

9 Henderson SE, Sudgen DA. Movement Assessment Battery for Children (MABC). London, UK: Psychological Corporation, 1992.

10 Bax MC. Terminology and classification of cerebral palsy. Dev Med Child Neurol 1964:6:295-7.

11 Geuze RH, Jongmans M, Schoemaker M, et al. Developmental coordination disorder. Hum Mov Sci 2001;20:1-5.

12 Blank R, Smits-Engelsman B, Polatajko H, et al. European Academy for Childhood Disability (EACD): recommendations on the definition, diagnosis and intervention of developmental coordination disorder (long version). Dev Med Child Neurol 2012:54:54-93.

13 Sugden DA, Chambers M, Utley A. Leeds Consensus Statement. 2006. http://www. dcd-uk.org/consensus.html (accessed 27 Mar 2012).

14 Williams J, Lee KJ, Anderson PJ. Prevalence of motor-skill impairment in preterm children who do not develop cerebral palsy: a systematic review. Dev Med Child Neurol 2010:52:232-7.

15 Yeh TF, Lin YJ, Lin HC, et al. Outcomes at school age after postnatal dexamethasone therapy for lung disease of prematurity. $N$ Engl I Med 2004;350:1304-13.

16 Al-Dahhan J, Jannoun L, Haycock GB. Effect of salt supplementation of newborn premature infants on neurodevelopmental outcome at 10-13 years of age. Arch Dis Child Fetal Neonatal Ed 2002;86:F120-3.

17 Missiuna C, Gaines R, McLean J, et al. Description of children identified by physicians as having developmental coordination disorder. Dev Med Child Neurol 2008:50:839-44.

18 De Vries LS, Van Haastert IL, Rademaker KJ, et al. Ultrasound abnormalities preceding cerebral palsy in high-risk preterm infants. J Pediatr 2004;144:815-20.

19 Spittle AJ, Cheong J, Doyle LW, et al. Neonatal white matter abnormality predicts childhood motor impairment in very preterm children. Dev Med Child Neurol 2011;53:1000-6.

20 Querne L, Berquin P, Vernier-Hauvette MP, et al. Dysfunction of the attentional brain network in children with developmental coordination disorder: a fMRI study. Brain Res 2008;1244:89-102.

21 Kashiwagi M, Iwaki S, Narumi Y, et al. Parietal dysfunction in developmental coordination disorder: a functional MRI study. Neuroreport 2009;20:1319-24.

22 Zwicker JG, Missiuna C, Harris SR, et al. Brain activation of children with developmental coordination disorder is different than peers. Pediatrics 2010;126: e678-86.

23 Zwicker JG, Missiuna C, Harris SR, et al. Brain activation associated with motor skill practice in children with developmental coordination disorder: an fMRI study. Int J Dev Neurosci 2011;29:145-52.

24 Zwicker JG, Missiuna C, Harris SR, et al. Developmental coordination disorder: a pilot diffusion tensor imaging study. Pediatr Neurol 2012;46:162-7.

25 Zwicker JG, Missiuna C, Boyd LA. Neural correlates of developmental coordination disorder: a review of hypotheses. J Child Neurol 2009;24:1273-81.

26 Limperopoulos C, Soul JS, Gauvreau K, et al. Late gestation cerebellar growth is rapid and impeded by premature birth. Pediatrics 2005;115:688-95.

27 Limperopoulos C, Bassan H, Gauvreau K, et al. Does cerebellar injury in premature infants contribute to the high prevalence of long-term cognitive, learning, and behavioral disability in survivors? Pediatrics 2007;120:584-93.

28 Volpe JJ. Cerebellum of the premature infant: rapidly developing, vulnerable, clinically important. J Child Neurol 2009;24:1085-104. 